\title{
Широкоапертурное полное поглощение терагерцовой волны в нанопериодической плазмонной структуре на основе графена
}

\author{
(C) О.В. Полищук*, В.С. Мельникова ${ }^{+}$, В.В. Попов*+• \\ * Саратовский фрилиал Института радиотехники и электроники \\ им. В.А. Котельникова Российской академии наук, \\ 410019 Саратов, Россия. \\ + Саратовский национальный исследовательский государственный университет, \\ 410012 Саратов, Россия \\ - Саратовский научный центр Российской академии наук, \\ 410028 Саратов, Россия \\ E-mail: polischuk.sfire@mail.ru, \\ popov_slava@yahoo.co.uk
}

(Получена 27 апреля 2016 г. Принята к печати 10 мая 2016 г.)

Теоретически исследован спектр поглощения терагерцового излучения периодическим массивом графеновых нанолент, расположенных на поверхности диэлектрика с высоким показателем преломления (терагерцовой призмы). Показано, что полное поглощение терагерцового излучения происходит в режиме полного внутреннего отражения терагерцовой волны от периодического массива графеновых нанолент на частотах плазменных колебаний в графене в широком диапазоне углов падения внешней терагерцовой волны, в том числе, при комнатной температуре.

\section{1. Введение}

Исследования графена, двумерного монослоя графита, в последнее время представляют большой интерес благодаря уникальным электронным свойствам этого материала, вытекающим из линейного вида (дираковского) бесщелевого энергетического спектра носителей заряда [1]. Графен обладает сильным плазмонным откликом на терагерцовых (ТГц) частотах, что обусловлено как высокой плотностью, так и малой коллективной эффективной массой свободных носителей заряда [2,3]. Использование плазменных колебаний носителей заряда в графене представляется привлекательным, так как позволяет сконцентрировать электромагнитное поле вблизи слоя графена и, таким образом, значительно повысить эффективность взаимодействия ТГц излучения с графеном. Это является важным как с точки зрения изучения физических свойств самого графена, так и для создания ТГц управляющих устройств на его основе.

Нанопериодические плазмонные структуры на основе графена представляют собой физически интересный объект. Так как длина возбуждаемой в такой структуре плазменной волны соизмерима с периодом структуры, данная структура образует планарный плазмонный кристалл. С другой стороны, поскольку пространственный период графеновой наноструктуры на 2-3 порядка величины меньше, чем длина волны падающего ТГц излучения, эту структуру можно рассматривать как планарную, резонансную ТГц метаповерхность, сильно связанную с ТГц излучением. В этом случае всю структуру можно характеризовать эффективным поверхностным импедансом, испытывающим резонанс на частотах возбуждения плазменных волн в графене, что позволяет найти усло- вия идеального согласования структуры с падающим ТГц излучением и определить условия наиболее эффективного возбуждения плазмонов в графене.

\section{2. Теоретическая модель}

В данной работе рассматривается периодический массив графеновых нанолент, расположенный на поверхности диэлектрика с высоким показателем преломления (ТГц призмы) (рис. 1). Внешняя ТГц волна падает на массив графеновых нанолент изнутри ТГц призмы под углом $\theta$. Направление периодичности массива графеновых нанолент лежит в плоскости падения плоской $p$-поляризованной ТГц волны. Электрическое поле

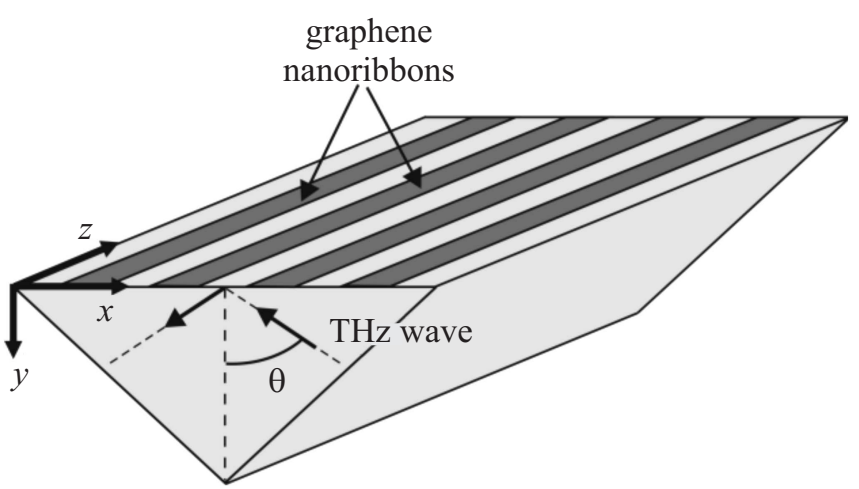

Рис. 1. Схематическое изображение структуры. Внешняя $p$-поляризованная ТГц волна падает на массив графеновых нанолент под углом $\theta$ изнутри призмы. Плоскость падения ТГц волны проходит поперек графеновых нанолент и совпадает с плоскостью чертежа. 
падающей волны $\mathbf{E}^{(0)} \exp [i(\mathbf{k r}-\omega t)]$ лежит в плоскости падения и имеет компоненты волнового вектора $k_{x 0}=-k_{0} \sin \theta$ и $k_{y 0}=-k_{0} \cos \theta$, где $k_{0}=\omega \sqrt{\varepsilon_{s}} / c, \omega-$ круговая частота, $c$ - скорость света в вакууме, $\varepsilon_{s}-$ диэлектрическая постоянная ТГц призмы. Задача о падении ТГц волны на периодический массив графеновых нанолент решается с использованием самосогласованного электродинамического подхода, аналогичного описанному в работе [4]. Решается полная система уравнений Максвелла с разложением искомых электрических и магнитных полей по плоским волнам. В рассматриваемой геометрии индуцированное электрическое поле имеет ненулевые $x$ - и $y$-компоненты, тогда как индуцированное магнитное поле имеет только ненулевую $z$-компоненту ( $p$-поляризация). Вследствие периодичности структуры в $x$-направлении, индуцированные электрические и магнитные поля можно представить в виде разложений Флоке-Блоха:

$$
\begin{aligned}
& H_{z}(x, y)=\sum_{m=-\infty}^{\infty} H_{z m}(y) \exp \left(-i q_{m} x\right), \\
& E_{x}(x, y)=\sum_{m=-\infty}^{\infty} E_{x m}(y) \exp \left(-i q_{m} x\right), \\
& E_{y}(x, y)=\sum_{m=-\infty}^{\infty} E_{y m}(y) \exp \left(-i q_{m} x\right),
\end{aligned}
$$

где

$$
\begin{aligned}
H_{z m}(y) & =\frac{1}{L} \int_{0}^{L} H_{z}(x, y) \exp \left(i q_{m} x\right) d x, \\
E_{x m}(y) & =\frac{1}{L} \int_{0}^{L} E_{x}(x, y) \exp \left(i q_{m} x\right) d x, \\
E_{y m}(y) & =\frac{1}{L} \int_{0}^{L} E_{y}(x, y) \exp \left(i q_{m} x\right) d x
\end{aligned}
$$

- амплитуды $m$-й фурье-гармоники соответственно магнитного и электрического полей и $q_{m}=k_{x 0}+2 \pi m / L$ $(m= \pm 1, \pm 2, \pm 3 \ldots)$.

Используя разложение (1) для компонент индуцированного электрического и магнитного полей, систему уравнений Максвелла в каждой среде можно записать в фурье-представлении. Стандартные электромагнитные граничные условия в плоскости $y=0$ (в плоскости массива графеновых нанолент) записываются в фурье-представлении как

$$
\begin{gathered}
E_{x m}^{(s)}(0)+\delta_{m 0} E_{x}^{(0)}=E_{x m}^{(a)}(0), \\
H_{z m}^{(s)}(0)+\delta_{m 0} H_{z}^{(0)}-H_{z m}^{(a)}(0)=\frac{4 \pi}{c} I_{x m}(0),
\end{gathered}
$$

где верхние индексы $a$ и $s$ относятся соответственно к окружающей среде (в рассматриваемом случае это вакуум) и подложке (ТГц призме), $\delta_{m 0}-$ символ
Кронекера. Величина $I_{x m}(0)$ представляет собой амплитуду $m$-й фурье-гармоники плотности поверхностного электрического тока в плоскости графеновых нанолент $y=0$. Используя граничные условия и уравнения Максвелла в каждой среде в фурье-представлении, после несложных, но достаточно громоздких алгебраических преобразований, получим следующее соотношение между $x$-компонентой электрического поля и плотностью поверхностного электрического тока в плоскости массива графеновых нанолент $y=0$ :

$$
E_{x m}^{(a)}(0)=Z_{m}\left[\frac{4 \pi}{c} I_{x m}(0)-\delta_{m 0} 2 E_{x}^{(0)}\right] .
$$

Величина $Z_{m}$ представляет собой эффективный поверхностный импеданс и задается формулой

$$
Z_{m}=1 /\left(\chi_{m}^{(s)}-\chi_{m}^{(a)}\right),
$$

где $\chi_{m}^{(a, s)}=\varepsilon_{a, s} k_{0} / \alpha_{m}^{(a, s)}$ и $\alpha_{m}^{(a, s)}= \pm \sqrt{q_{m}^{2}-k_{0}^{2} \varepsilon_{a, s}}$. Знаки перед радикалом в выражениях для поперечных волновых чисел $\alpha_{m}^{(a)}$ и $\alpha_{m}^{(s)}$ выбраны таким образом, что они соответствуют убегающим волнам при $y \rightarrow \pm \infty$ (так называемое условие излучения [5]) для $q_{m}^{2}<k_{0}^{2} \varepsilon_{a, s}$ (в этом случае $\alpha_{m}^{(a)}$ и $\alpha_{m}^{(s)}-$ мнимые величины) и эванесцентным волнам, затухающим на бесконечности при $y \rightarrow \pm \infty$ для $q_{m}^{2}>k_{0}^{2} \varepsilon_{a, s}$ (в этом случае $\alpha_{m}^{(a)}$ и $\alpha_{m}^{(s)}$ - реальные величины).

Используя закон Ома в графене совместно с выражением для плотности электрического тока, полученного из электромагнитных граничных условий, получаем интегральное уравнение для $x$-компоненты плотности электрического тока в плоскости массива графеновых нанолент:

$$
\frac{1}{L} \int_{0}^{w} \bar{Z}\left(x, x^{\prime}\right) I_{x}\left(x^{\prime}\right) d x^{\prime}=2 E_{x}^{(0)} Z_{0} \sigma_{\mathrm{Gr}}(\omega),
$$

где

$$
\bar{Z}\left(x, x^{\prime}\right)=\sum_{m=-\infty}^{\infty}\left(1+\frac{4 \pi}{c} \sigma_{\mathrm{Gr}}(\omega) Z_{m}\right) \exp \left[i q_{m}\left(x-x^{\prime}\right)\right]
$$

$Z_{0}=1 /\left(\chi_{0}^{(s)}-\chi_{0}^{(a)}\right)$ и $\sigma_{\mathrm{Gr}}(\omega)-$ комплексная динамическая проводимость графена. Уравнение (4) представляет собой интегральное уравнение Фредгольма первого рода с ядром $\bar{Z}\left(x, x^{\prime}\right)$.

Полученное интегральное уравнение решалось численно методом Галеркина с разложением искомой функции ( $x$-компоненты плотности электрического тока) в графеновых нанолентах $I_{x}(x)=\sum_{n=1}^{\infty} a_{n} P_{n}(x)$ по ортогональным полиномам Лежандра $P_{n}(x /(w / 2)-1)$ на интервале $0 \leq x \leq w$ с неизвестными константами $a_{n}$.

Индуцированные электрические и магнитные поля в любой точке структуры могут быть найдены с помощью 


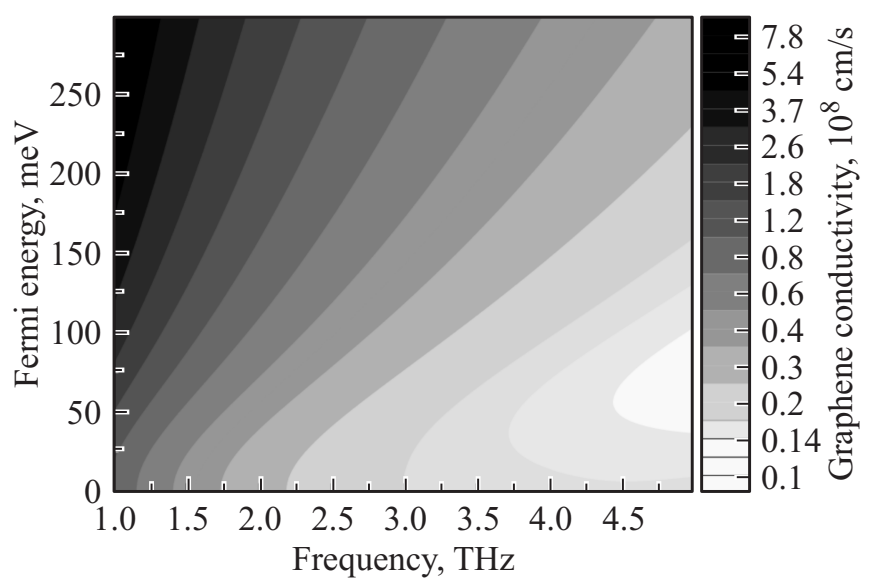

Рис. 2. Контурная карта действительной части проводимости графена в зависимости от частоты и величины энергии Ферми при $\gamma=10^{12} \mathrm{c}^{-1}$ и $T=300 \mathrm{~K}$.

выражения (3) и уравнений Максвелла в каждой из сред. Выражение для комплексной амплитуды продольного электрического поля в плоскости графеновых нанолент имеет вид

$$
\begin{aligned}
E_{x}(x, 0)= & \frac{w}{L} \sum_{n=1}^{\infty} a_{n}(-i)^{n} \sum_{m=-\infty}^{\infty} \exp \left(i q_{m} x\right) Z_{m} J_{n}\left(\frac{q_{m} w}{2}\right) \\
& \times \exp \left(-i q_{m} \frac{w}{2}\right)-2 Z_{0} E_{x}^{(0)}
\end{aligned}
$$

где $J_{n}\left(q_{m} w / 2\right)$ - сферические функции Бесселя.

Длина волны резонансной плазмонной моды, возбуждаемой в графене падающей ТГц волной, определяется периодом $L$ массива и шириной $w$ графеновых нанолент. Поскольку длина волны плазмона в графене намного (на 2-3 порядка) короче, чем длина электромагнитной волны на той же частоте [6,7], для возбуждения плазмонных резонансов в структуре необходимо выполнение условия $L \ll 2 \pi / k_{0}$. В этом случае только нулевые фурье-гармоники $(m=0)$ соответствуют бегущим волнам, излучаемым в окружающую среду и в подложку (ТГц призму), а все фурье-гармоники более высокого порядка являются затухающими волнами, убывающими при $y \rightarrow \pm \infty$. Следовательно, коэффициенты отражения $R$ и пропускания $T$ исследуемой структуры в дальнем поле могут быть вычислены соответственно как $R=\left|E_{x 0}(0)\right|^{2} \sqrt{\varepsilon_{s}} /\left|E^{(0)}\right|^{2}$ и $T=\left|E_{x 0}(0)\right|^{2} /\left|E^{(0)}\right|^{2}$. Коэффициент поглощения рассчитывается как $A=P_{\mathrm{abs}} / P_{\mathrm{in}}$, где $P_{\text {in }}$ - плотность мощности падающей ТГц волны и

$$
P_{\mathrm{abs}}=\frac{1}{2 L} \operatorname{Re}\left[\sigma_{\mathrm{Gr}}(\omega)\right] \int_{-L / 2}^{L / 2}\left|E_{x}(x, 0)\right|^{2} d x
$$

- поглощенная ТГц мощность на периоде структуры.

Отклик графена описывается комплексной динамической проводимостью [8]:

$$
\sigma_{\mathrm{Gr}}(\omega)=\sigma_{\mathrm{intra}}(\omega)+\sigma_{\mathrm{inter}}(\omega)
$$

где

$$
\sigma_{\text {intra }}(\omega)=\sigma_{0} \frac{8 i k_{\mathrm{B}} T}{\pi \hbar(\omega+i \gamma)} \ln \left[2 \cosh \left(\frac{E_{\mathrm{F}}}{2 k_{\mathrm{B}} T}\right)\right]
$$

и

$$
\begin{gathered}
\sigma_{\text {inter }}(\omega)=\sigma_{0}\left\{G\left(\frac{\hbar \omega}{2}\right)+\frac{4 i \hbar \omega}{\pi} \int_{0}^{\infty} \frac{G(\xi)-G(\hbar \omega / 2)}{(\hbar \omega)^{2}-4 \zeta^{2}} d \xi\right\}, \\
G(\xi)=\frac{\sinh \left(\xi / k_{\mathrm{B}} T\right)}{\cosh \left(E_{\mathrm{F}} / k_{\mathrm{B}} T\right)+\cosh \left(\xi / k_{\mathrm{B}} T\right)} .
\end{gathered}
$$

Здесь $\sigma_{0}=e^{2} / 4 \hbar, E_{\mathrm{F}}$ - энергия Ферми, температура $T$ равна $300 \mathrm{~K}, \omega$ - круговая частота падающей ТГц электромагнитной волны, $e-$ заряд электрона, $k_{\mathrm{B}}-$ постоянная Больцмана и $\hbar-$ приведенная постоянная Планка. Первое слагаемое в формуле (5) описывает отклик в форме Друде с участием внутризонных процессов, описываемых феноменологической скоростью внутризонного рассеяния электронов $\gamma$. Второе слагаемое описывает межзонные переходы в графене. Реальная часть проводимости (5), ответственная за диссипацию энергии и учитывающая оба механизма рассеяния, представлена на рис. 2.

Численные расчеты выполнены для массива графеновых полосок с периодом $L=2$ мкм и шириной графеновых полосок $w=1$ мкм, расположенных на поверхности кремниевой подложки, с диэлектрической постоянной $\varepsilon_{s}=11.7$. Феноменологическая скорость внутризонного рассеяния электронов $\gamma$ полагается равной $10^{12} \mathrm{c}^{-1}$.

\section{3. Результаты расчетов и обсуждение}

Численно рассчитанные спектры ТГц поглощения в зависимости от частоты и величины энергии Ферми представлены на рис. 3.

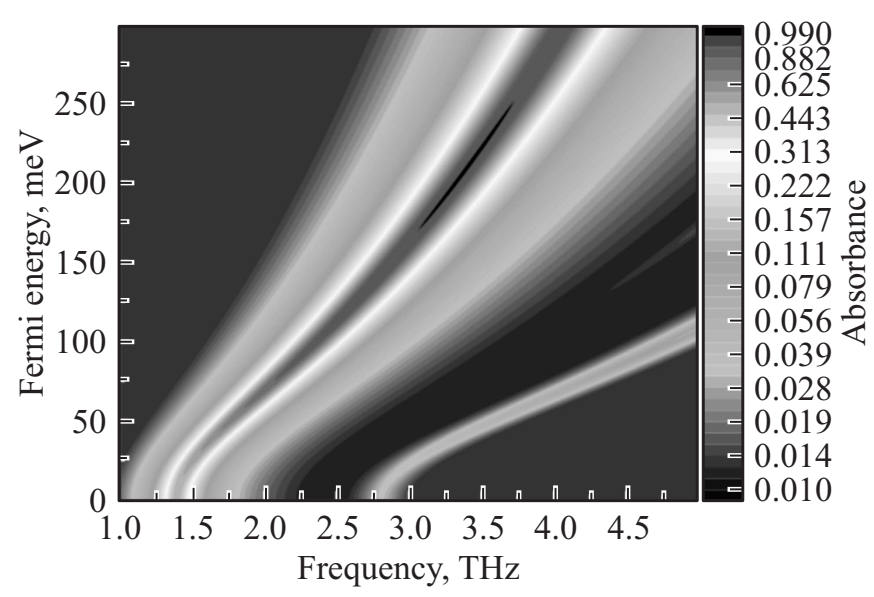

Рис. 3. Спектр ТГц поглощения для основного и высших плазмонных резонансов в режиме полного внутреннего отражения для массива графеновых нанолент шириной 1 мкм с периодом 2 мкм как функция частоты и величины энергии Ферми при угле падения $\theta=30^{\circ}$. 
Расчеты выполнены для реалистичных параметров массива графеновых нанолент при комнатной температуре и $\gamma=10^{12} \mathrm{c}^{-1}$. В этом случае поглощение ТГц волны вызвано преимущественно внутризонными процессами, описываемыми феноменологической скоростью внутризонного рассеяния электронов $\gamma$. Поглощение ТГц волны, связанное с межзонной генерацией электронно-дырочных пар в графене существенно только при низких значениях энергии Ферми $\left(E_{\mathrm{F}} \sim \hbar \omega / 2\right)$ (см. рис. $4, a)$ и уменьшается с увеличением энергии Ферми, в соответствии с поведением действительной части проводимости графена при нулевой скорости внутризонного рассеяния электронов $\gamma$ (см. рис. $4, b)$.

Рассчитанные спектры ТГц поглощения в зависимости от частоты и угла падения внешней волны при различных значениях величины энергии Ферми приведены на рис. 5. Из рис. 5 видно, что в режиме полного внутреннего отражения $\theta>\theta_{R}$, где $\theta_{R}-$ угол полного внутреннего отражения $\left(\theta_{R} \approx 17^{\circ}\right.$ при $\left.\varepsilon_{2}=11.7\right)$, реализуется эффект полного поглощения падающего
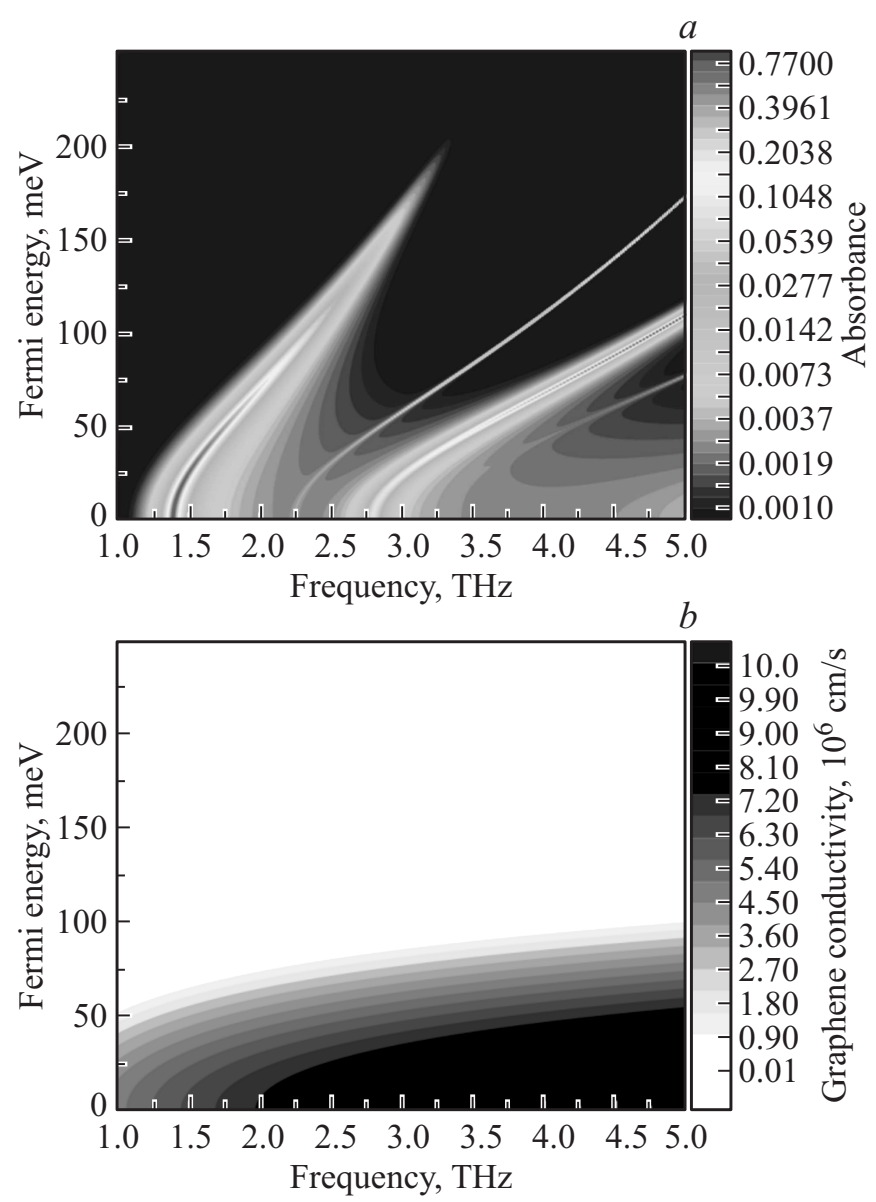

Рис. 4. Спектр ТГц поглощения для основного и высших плазмонных резонансов в режиме полного внутреннего отражения для массива графеновых нанолент шириной 1 мкм с периодом 2 мкм как функция частоты и величины энергии Ферми для угла падения $\theta=30^{\circ}$ и $\gamma=0(a)$. Контурная карта действительной части проводимости графена в зависимости от частоты и величины уровня Ферми при $\gamma=0(b)$.

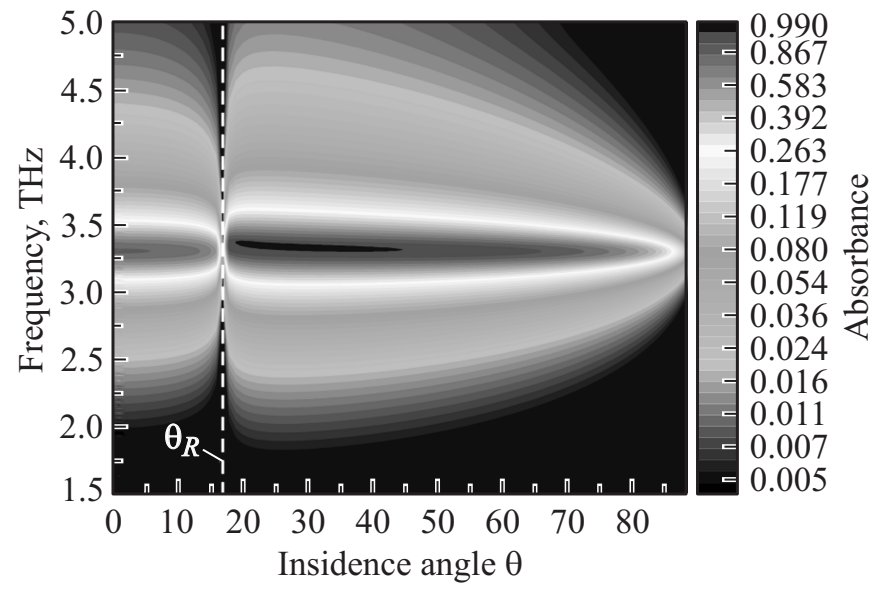

Рис. 5. Спектр ТГц поглощения для основного плазмонного резонанса в массиве графеновых нанолент шириной 1 мкм с периодом 2 мкм как функция величины угла падения $\theta$ и частоты для величины энергии Ферми $E_{\mathrm{F}}=200$ мэВ.

ТГц излучения на частотах плазменных колебаний в графене в широком диапазоне углов падения (узкий темный лепесток на рис. 5). Заметим что эффект полного поглощения падающего ТГц излучения на частотах плазменных колебаний в графене возможен при соблюдении условия баланса диссипативных и радиационных потерь [9]. Диссипативные потери определяются феноменологической скоростью внутризонного рассеяния электронов $\gamma$, а радиационные потери зависят от концентрации свободных носителей заряда и определяются положением уровня Ферми.

Резонансная частота плазменных колебаний в графене может варьироваться как при изменении положения уровня Ферми (рис. 3), так и ширины графеновых нанолент.

\section{4. Заключение}

В работе теоретически исследован эффект полного поглощения терагерцовой волны периодическим массивом графеновых нанолент, расположенным на поверхности диэлектрика с высоким показателем преломления (ТГц призмы). Показано, что эффект полного поглощения терагерцового излучения может быть достигнут в режиме полного внутреннего отражения ТГц волны от периодического массива графеновых нанолент на частотах плазменных колебаний в графене в широком диапазоне углов падения внешней ТГц волны, в том числе, при комнатной температуре. Полученные результаты могут быть использованы для создания компактных планарных частотно перестраиваемых наноразмерных ТГц управляющих устройств на основе графена.

Работа выполнена при поддержке гранта РФФИ № 16-02-00814. 


\section{Список литературы}

[1] K.S. Novoselov, A.K. Geim, S.V. Morozov, D. Jiang, M.I. Katsnelson, I.V. Grigorieva, S.V. Dubonos, A.A. Firsov. Nature, 438, 197 (2005)

[2] J. Chen, M. Badioli, P. Alonso-González, S. Thongrattanasiri, F. Huth, R. Hillenbrand, F. Koppens. Nature, 487, 77 (2012).

[3] Z. Fei, A.S. Rodin, G.O. Andreev, W. Bao, A.S. McLeod, M. Wagner, L.M. Zhang, Z. Zhao, M. Thiemens, G. Dominguez, M.M. Fogler, A.H. Castro Neto, C.N. Lau, F. Keilmann, D.N. Basov. Nature, 487, 82 (2012).

[4] Д.В. Фатеев, В.В. Попов, M.S. Shur. ФТП, 44 (11), 1455 (2010).

[5] L.A. Weinstein. Open Resonators and Open Waveguides (Golem, N. Y., 1969).

[6] J. Chen, M. Badioli, P. Alonso-González, S. Thongrattanasiri, F. Huth, J. Osmond, M. Spasenović, A. Centeno, A. Pesquera, P. Godignon, A.Z. Elorza, N. Camara, F.I. García de Abajo, R. Hillenbrand, F.H.L. Koppens. Nature, 487, 77 (2012).

[7] Z. Fei, A.S. Rodin, G.O. Andreev, W. Bao, A.S. McLeod, M. Wagner, L.M. Zhang, Z. Zhao, M. Thiemens, G. Dominguez, M.M. Fogler, A.H. Castro Neto, C.N. Lau, F. Keilmann, D.N. Basov. Nature, 487, 82 (2012).

[8] L.A. Falkovsky, A.A. Varlamov. Eur. Phys. J. B, 56, 281 (2007).

[9] V.V. Popov, O.V. Polischuk, T.V. Teperik, X.G. Peralta, S.J. Allen, N.J.M. Horing, M.C. Wanke. J. Appl. Phys., 94, 3556 (2003).

Редактор А.Н. Смирнов

\section{Wide-aperture total absorption of terahertz radiation by plasmonic nanoperiodical graphene-based structure}

O.V. Polischuk*, V.S. Melnikova ${ }^{+}$, V.V. Popov ${ }^{*+\bullet}$

+ Kotelnikov Institute of Radio Engineering and Electronics (Saratov Branch),

Russian Academy of Sciences,

410019 Saratov, Russia

+ National Research Saratov State University,

410012 Saratov, Russia

- Saratov Scientific Center

of the Russian Academy of Sciences,

410028 Saratov, Russia

Abstract The absorbance spectrum of terahertz radiation by periodic array of graphene nanoribbons located on the surface of dielectric substrate with a high refractive index (terahertz prism) is studied theoretically. The effect of the total absorption of terahertz radiation takes place in the total internal reflection regime from a periodic array of graphene nanoribbons at frequencies of plasma oscillations in graphene. It is shown that the total absorption of terahertz radiation can be obtained at a wide range of the angles of incidence of the external terahertz wave even at room temperature. 